\title{
Müllerian Adenosarcoma of the Vaginal Stump: A Case Report and Literature Review
}

\author{
Juan Li' ${ }^{1}$ Zhenling Duan ${ }^{*} \#$, Ma Jing3, Xiaotao Jing2, Li Bian ${ }^{4}$ \\ ${ }^{1}$ Gynaecology Department, Dali Bai Minority Autonomous Prefecture People's Hospital, Yunnan, China \\ ${ }^{2}$ Gynaecology Department, First Affiliated Hospital of Kunming Medical University, Yunnan, China \\ ${ }^{3}$ Obstetrics Department, First Affiliated Hospital of Kunming Medical University, Yunnan, China \\ ${ }^{4}$ Pathology Department, First Affiliated Hospital of Kunming Medical University, Yunnan, China \\ Email: *zhenlingduan@126.com
}

How to cite this paper: Li, J., Duan, Z.L., Jing, M., Jing, X.T. and Bian, L. (2018) Müllerian Adenosarcoma of the Vaginal Stump: A Case Report and Literature Review. Journal of Cancer Therapy, 9, 914-924. https://doi.org/10.4236/jct.2018.911076

Received: August 12, 2017

Accepted: November 23, 2018

Published: November 26, 2018

Copyright () 2018 by authors and Scientific Research Publishing Inc. This work is licensed under the Creative Commons Attribution International License (CC BY 4.0).

http://creativecommons.org/licenses/by/4.0/

\begin{abstract}
Müllerian adenosarcomas are rare mixed tumors with low malignant potential, and occur mainly in the uterus. Primary adenosarcomas arising in vaginal endometriosis are even more rarely reported. We report a very rare case with Müllerian adenosarcoma arising from vaginal endometriosis. A 43-year-old nulliparous woman has a history of endometriosis. Due to two parallel fistulas in cervix, severe endometriosis and adenosarcoma, she has undergone radical and multiple surgeries. After the surgery, persistent vaginal vault masses were noticed, and eight resections of the polyps at vaginal stump were performed. For the first six resections, all the pathological examinations showed endometriosis. And the last two resections demonstrated vaginal adenosarcoma. Transition between Müllerian adenosarcoma and endometriosis was gradual. Finally, adenosarcoma associated with endometriosis was diagnosed from recurrent vaginal masses. To our knowledge, this report is the sixth case that primary adenosarcomas arising in vaginal endometriosis. But chemo-therapy and radiation therapy were not taken and the patient remained in a good condition throughout the 9-year follow-up period. In addition, we present a review of the literature. The diagnosis and treatment of the tumor are also discussed.
\end{abstract}

\section{Keywords}

Endometriosis, Vaginal Stump, Müllerian Adenosarcoma, Case Report

\section{Introduction}

Müllerian adenosarcoma (MA) is a rare mixed and low malignant tumor of female genital system, and is a biphasic differential tumor that is composed of ad${ }^{\#}$ co-first author. 
mixed benign epithelial and sarcomatoid mesenchymal components. Difficulties are commonly encountered in diagnosis and differential diagnosis because of the rarity. Müllerian adenosarcomas are rare tumors with non-specific clinical features, and this forms a circumstance that poses significant challenges to the clinical practice or even delayed treatment when women presented with complaints of abdominal pain and irregular vaginal bleeding from the endometriosis postoperatively. Endometriosis has the potential to develop into malignant form. Postmenopausal women or young girls who have been repeatedly treated for endometriosis, adenosarcomas should be considered. The pathological feature of adenosarcoma is called "cuffing structure", atypical hyperplasia of the cellular stromas that surround the glandular component, like dense periglandular cuffs. To the best of our knowledge, our case is the sixth case that primary adenosarcomas arising in vaginal endometriosis and it is the first case living without chemo-therapy or radiotherapy. The aim of this report is to analyze the histological features, diagnosis and treatments of the tumor. Finally, regular clinic follow-ups are necessary.

\section{Case Report}

A 43-year-old nulligravida female patient was hospitalized on October $20^{\text {th }}, 2009$, complaining of irregular vaginal bleeding for more than 1 month. She had endometriosis and has undergone supra-cervical hysterectomy. After the surgery, the patient had recurrent vaginal stump polyps for 10 years. At last, the patient's histological features support the diagnosis of vaginal adenosarcoma.

The patient has menarche since 12. Her menstrual cycle lasts for 5 - 7 days every 28 - 31 days. The patient did not have dysmenorrhea. In November 1989 (when she was 22 years old), the patient had experienced dysmenorrhea that has interfered with her work and life seriously. On December $10^{\text {th }}, 1990$, the patient was diagnosed as appendicitis and ovarian cyst, and had appendecetomy and left ovarian chocolate cystectomy at a local hospital. Soon after the surgery, her dysmenorrhea was aggravating, and the patient was diagnosed as endometriosis. She has gone through some symptomatic treatment, but the therapy only slightly alleviated the symptom.

In March 1998, the patient was found to have multiple polys in the vaginal posterior fornix and a fistula that was at the posterior fornix and extended to the pelvic cavity. She had surgical therapy in The First Affiliated Hospital of Kunming Medical University. During her surgery, adhesions of peritoneum, omentum and sigmoid colon were noticed. Her uterus has also been found enlarged to the size of two gestational months, and was firm and inactive. Some small yellow exudative vesicles were identified on the surface of the uterus. Adhesions have also been found between the rectum and posterior uterine wall, which totally blocked the pouch of douglas. A $7 \mathrm{~cm} \times 6 \mathrm{~cm} \times 6 \mathrm{~cm}$ cyst was found on right

adnexa. Thus, right integrated tubo-ovarian abscess was confirmed. Once the adhesions of posterior uterine wall were separated, the pouch of douglas was 
exposed. Moreover, two fistulas were identified. They were at the sizes of $2.5 \mathrm{~cm}$ and $2 \mathrm{~cm}$, and located close to the cervix. It's hard to repair the fistulas without uterus resection since the uterus was relatively big. Therefore hysterectomy was considered. The hysterectomy therapy was informed to the family and consent was obtained. Finally, the patient underwent total hysterectomy, right salpingectomy, evacuation and fistulization of the abscess and vaginal fistula repair. The pathological examination revealed: 1) Posterior fornix, vagina, cervix, omentum and myometrium endometriosis; 2) Pelvic vaginal fistula.

In June 1999, the patient had a vaginal stump neoplasm resection, and the biopsy showed "inflammatory polyp". In March 2001, the patient was found having a rapidly enlarged mass at a size of $8.4 \mathrm{~cm} \times 5.5 \mathrm{~cm} \times 9.7 \mathrm{~cm}$ in the pelvis and a fistula at the vaginal apex. Ultrasound-guided paracentesis was performed. The mass was shrunk to $3.5 \mathrm{~cm} \times 3.4 \mathrm{~cm}$. The extracted fetid pus revealed escherichia coli infection without acid-fast bacilli. The patient received antibiotic treatment.

In February 2006, the patient presented with irregular neoplasm of the vaginal stump and pelvic mass, and a biopsy revealed endometriosis with chronic nonspecific inflammation. By the end of 2006, grossly, the vaginal stump was filled with polypoid and stripe-type masses measuring $4.0 \mathrm{~cm} \times 3.0 \mathrm{~cm} \times 2.0 \mathrm{~cm}$, and the patient was admitted to the Peking Union Medical College Hospital. The polyps were removed and the pathological examination demonstrated inflammatory polyp with endometriosis, and diagnosed as "actinomycosis". After the surgery, persistent vaginal vault masses were noticed, and three resections of the polyps at vaginal stump were performed in April, May and June of 2008. The pathological examination showed endometriosis with chronic inflammation (Figure 1). August $15^{\text {th }}, 2008$, the patient underwent exploratory laparoscopy and vaginal stump fistula suture in the hospital. During the operation, a pelvic encapsulated effusion and a fistula on the left side of vaginal stump were identified.

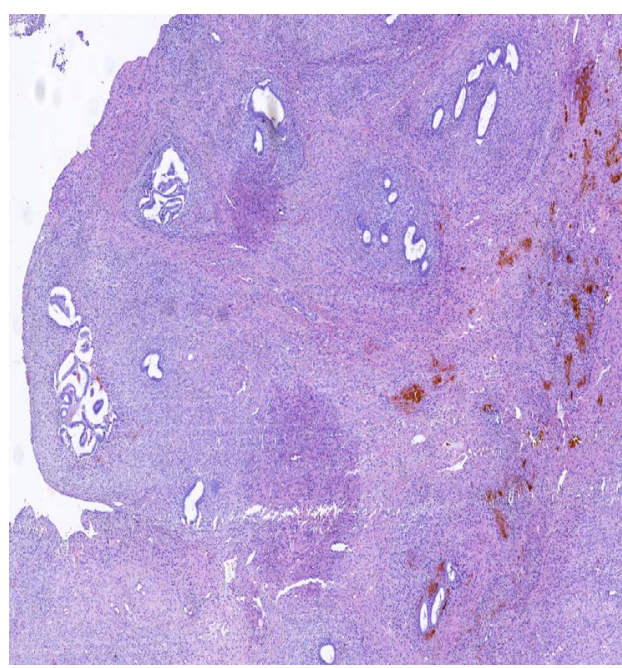

Figure 1. Endometriosis in vaginal stump biopsy. HE, $\times 4$. 
September $11^{\text {th }} 2009$, a solid and heterogeneous mass without envelope and extended to the vagina was identified in pelvis by transvaginal ultrasound. And 4 $\mathrm{cm} \times 3 \mathrm{~cm}$ vaginal mass was found. The patient underwent a resection of vaginal neoplasm and the pathological examination demonstrated vaginal adenosarcoma arising in endometriosis. On microscopic examination, the stromal mitotic rate was reported as 1 - 2 per 10 high-power fields (Figure 2).

MA is a rare tumor with heterogeneity and non-specific clinical features. It is difficult to be diagnosed at the early stage. The definitive diagnosis is relied on the postoperative pathological examination, and required the presence of at lease one of the following features: two or more stromal mitoses per $10 \mathrm{HPFs}$, mild atypia and active stromal cell proliferation [1]. However, adenosarcoma has been reported in the cases in which none of the above criteria has been reached, and even with a negligible mitotic rate. Current studies have indicated that the distinctive periglandular cuffs of the cellular stroma could be of diagnostic significance [2]. In addition, immunohistochemistry staining can be used to distinguish the sources of the sarcoma components. There was $1-2$ stromal mitotic figures per 10 high-power fields $(1-2 / 10 \mathrm{HPF})$ and mild nuclear atypia were identified (Figure 3). Benign endometriotic glands are embedded in a cellular stroma with polyp-like features. The periglandular stromal cells are dense and nuclear atypia, ranged moderate differentiation. Cuffing of cellular stroma around some of the glands was also identified (Figure 4). Foci of endometriosis were also identified in some areas. These histological features support the diagnosis of adenosarcoma arising in endometriosis.

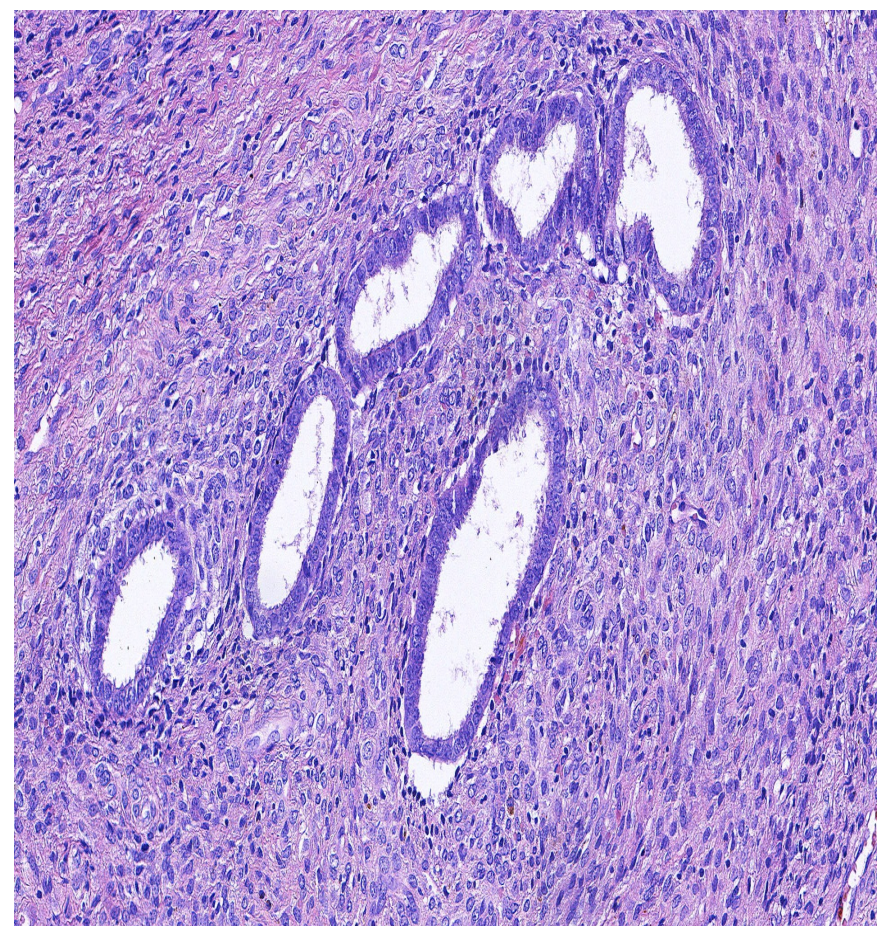

Figure 2. Low-power examination to the adenosarcoma. Mitotic figures around endometriotic glands. HE, $\times 20$. 


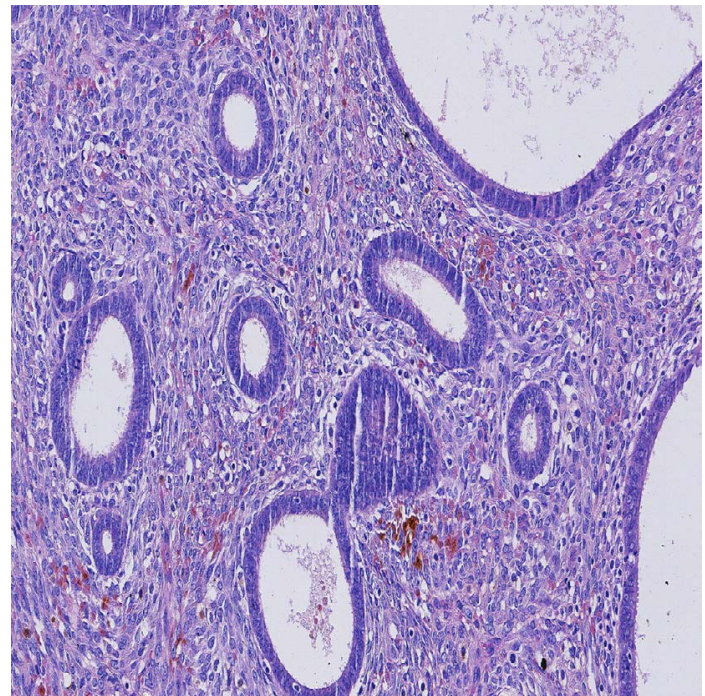

Figure 3. Low-power examination. Cellular stroma with mild nuclear atypia. HE, $\times 20$.

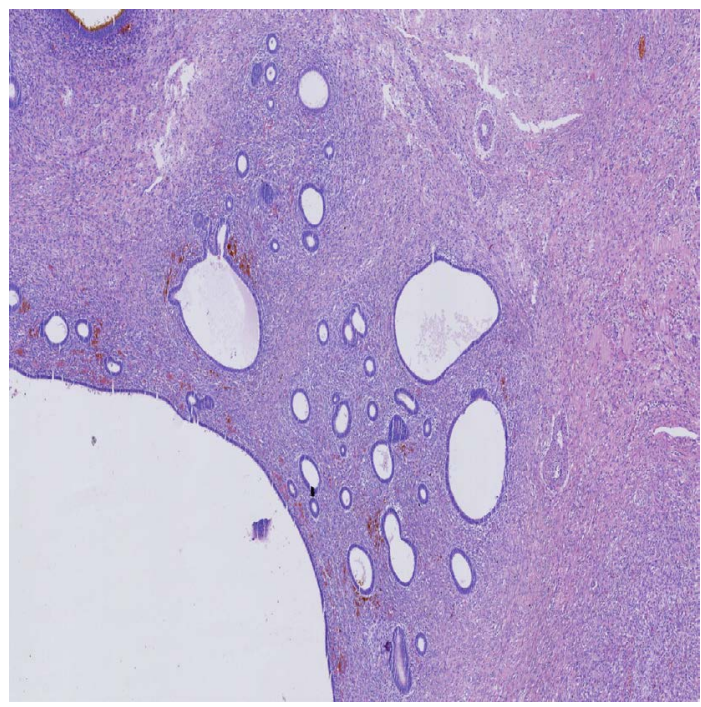

Figure 4. Adenosarcoma with endometriotic glands and periglandular cuff of increased stromal cellularity. $\mathrm{HE}, \times 4$.

On October $28^{\text {th }}, 2009$, the patient underwent surgery because of adenosarcoma of vaginal stump, right ovarian cyst, pelvic vaginal fistula and recurrent neoplasm at vaginal stump. The pelvic examination found a $3 \mathrm{~cm} \times 3 \mathrm{~cm}$ neoplasm at the vaginal apex, and it was friable and tended to bleed. Palpation identified a $10 \mathrm{~cm}$ pelvic mass with undefined edges. The patient's tumor markers were within normal ranges. The ultrasonography revealed left mild hydronephrosis and the left ureter upside expansion. After consulting with Oncological Surgery and Urological Surgery, a left double-J stent was placed pre-operation and a combined surgery of partial colpectomy, pelvic mass resection, bilateral adnexectomy, left bladder repair, partial small intestine resection with end-to-end anastomosis, segmental sigmoidectomy with end-to-end anastomosis, right ureterovesical reimplantation plus bladder repair and pelvic adhesiolysis were per- 
formed. During the operation, the patient was noted to have serious intra-pelvic adhesions, and a pelvic mass adhered to the sigmoid colon and the left colon was noticed. Thus, segmental resection of the adherent sigmoid colon with end-to-end anastomosis was performed. The vaginal mass was found infiltrated through the fistula of vaginal stump, and adhered to the pelvic mass. The $2.5 \mathrm{~cm}$ long vaginal stump was removed. In addition, a pelvic adhesive mass was found attached to the left bladder. The adhesion was separated and bladder repair was performed. Finally, the patient was also noted to have significant adhesions and fibrosis between the pelvic segments and the right ureter, especially at the entrance where the ureter connects the bladder. $2 \mathrm{~cm}$ of the previous segment of the right ureter was resected and right ureterovesical replantation was conducted. A right double-J stent was placed at the same time. After the surgery, the pathological examination demonstrated endometriosis and adenosarcoma associated with endometriosis in the vaginal stump. No tumor metastases were identified in the small intestine, rectum sigmoid colon, right ureter and mesenteric lymph nodes.

In January 2010, three months after the operation, the double-J stent was removed. The wound was healed well, and no urethral fistula or fecal fistula was identified. Chemo-therapy and radiation therapy were not taken. The patient was followed up regularly for 9 years. Her serum CA-125 and Magnetic Resonance Imaging examination were negative. No visible vaginal lesions were found. Chemo-therapy and radiotherapy were not taken and the patient remained in a good condition throughout the 9-year follow-up period.

\section{Discussions}

Müllerian adenosarcoma (MA), a rare mixed tumor of female genital system, is a biphasic differential tumor that is composed of admixed benign epithelial and sarcomatoid mesenchymal components. MA is accounted for only $8 \%$ of all uterine sarcomas. Although MA develops mostly in postmenopausal women, it can occur in teenage as well. Endometrium was the primary site in patients with MA arising in endometriosis. However, cases with extra-uterine sites such as in the ovary, peritoneum, intestinal canal and vagina have also been reported, with vagina as the mostly rare case. So far only five cases of primary adenosarcoma arising in vaginal endometriosis have been reported in the literature [3] [4] [5] [6] [7] (Table 1), and our current case is another one.

In the five reported cases, the patients aged from 34 to 56 years old, all have a history of endometriosis. It took 3 - 10 years from the original diagnosis of endometriosis to vaginal adenosarcoma. Three patients had aggressive and recurrent endometriosis. Another case [5] was a 28-year-old nulligravida patient, and it took 53 months to identify the primary finding of adenosarcoma. This patient underwent hysterectomy and right ovarian cystectomy due to severe endometriosis. In addition, she received several pelvic surgeries including rectovaginal septum nodules resection, partial colectomy, right adnexectomy and four excisions of vaginal lesions due to recurrent vaginal neoplasms. Malignant changes 
Table 1. Reported cases of vaginal Mullerian adenosarcoma.

\begin{tabular}{|c|c|c|c|c|c|c|c|c|}
\hline Reference & $\begin{array}{c}\text { Age } \\
\text { (year) }\end{array}$ & $\begin{array}{l}\text { Size } \\
(\mathrm{cm})\end{array}$ & $\begin{array}{l}\text { Interval } \\
\text { time } \\
\text { (year) }\end{array}$ & $\begin{array}{l}\text { Pathological } \\
\text { course }\end{array}$ & $\begin{array}{l}\text { Stromal mitotic } \\
\text { figures }(/ 10 \mathrm{HF})\end{array}$ & Treatment category & Follow-up & $\begin{array}{c}\text { Presence of } \\
\text { metastasis }\end{array}$ \\
\hline $\begin{array}{l}\text { Judson, } \\
\text { et al. [1] }\end{array}$ & 42 & 6 & 4 & $\begin{array}{l}\text { Vaginal EM, } \\
\text { Vaginal MA }\end{array}$ & 3 & $\begin{array}{l}6 \text { courses of PC and external } \\
\text { beam and brachyradiotherapy }\end{array}$ & $\begin{array}{l}\text { Regular and } \\
\text { free of disease }\end{array}$ & None \\
\hline $\begin{array}{l}\text { Anderson, } \\
\text { et al. [2] }\end{array}$ & 46 & 10 & 3.7 & $\begin{array}{l}\text { Vaginal EM, } \\
\text { Vaginal MA }\end{array}$ & 4 & $\begin{array}{l}\text { Extetnal radiotherapy and } \\
\text { interstitial brachytherapy }\end{array}$ & $\begin{array}{l}\text { Regular and } \\
\text { free of disease }\end{array}$ & None \\
\hline $\begin{array}{l}\text { Liu, } \\
\text { et al. [3] }\end{array}$ & 56 & 16 & 3 & $\begin{array}{l}\text { Vaginal EM, } \\
\text { Vaginal MA }\end{array}$ & occasional & $\begin{array}{l}\text { PI chemotherapy and } \\
\text { pelvic adiotherapy }\end{array}$ & $\begin{array}{l}\text { Regular and } \\
\text { free of disease }\end{array}$ & None \\
\hline $\begin{array}{l}\text { Toyoshima, } \\
\text { et al. [4] }\end{array}$ & 52 & 11 & 10 & $\begin{array}{l}\text { Vaginal EM, } \\
\text { Vaginal MA }\end{array}$ & several & $\begin{array}{l}3 \text { ourses of } \mathrm{CP} \text { and } \\
1 \text { course of } \mathrm{PC}\end{array}$ & $\begin{array}{l}\text { Died } 9 \text { months } \\
\text { after surgery }\end{array}$ & Lungs \\
\hline $\begin{array}{l}\text { Han, } \\
\text { et al. [5] }\end{array}$ & 34 & 6 & 4.5 & $\begin{array}{l}\text { Vaginal EM, } \\
\text { Adenofibroma } \\
\text { with part of } \\
\text { low-grade MA }\end{array}$ & occasional & $\begin{array}{l}3 \text { courses of PVP } \\
\text { and } 9 \text { courses of PEI }\end{array}$ & $\begin{array}{l}\text { Regular and no } \\
\text { recurrence }\end{array}$ & None \\
\hline $\begin{array}{l}\text { Li and Duan, } \\
\text { et al. (current case) }\end{array}$ & 43 & 4 & 10 & $\begin{array}{l}\text { Vaginal EM, } \\
\text { Vaginal MA }\end{array}$ & $1-2$ & $\begin{array}{l}\text { No chemotherapy } \\
\text { and radiotherapy }\end{array}$ & $\begin{array}{l}\text { Regular and no } \\
\text { recurrence }\end{array}$ & None \\
\hline
\end{tabular}

MA: Mullerian Adenosarcoma; EM: Endometriosis; PC: Paclitaxel and Carboplatin; PI: Cisplatin and Ifosfamide; CP: Carboplatin and Pirarubicin; PVP: Cisplatin, Vincristine and Pingyangmycin; PEI: Cisplatin, Epirubicin and Iphosphamide.

were evident by the pathological examination at each stage, progressing from endometriosis to MA in vaginal stump, and to MA with localized low-grade adenosarcoma. In our case, the patient is a 43-year-old nulligravida women, her diagnosis of vaginal adenosarcoma arising in endometriosis took about 10 years. During this period she presented with repeatedly vaginal mass. Our case meets the majority characteristics of endometriosis malignant transformation. Vaginal MA is a very rare tumor. Up to now, only 5 cases reported in the literature. Ours would be counted as the $6^{\text {th }}$. All patients in these six cases had a history of endometriosis. The malignant transformation has been reported approximately $0.7 \%-1.0 \%$ of women with endometriosis, and the majority is epithelial tumor. However, malignant mesenchymal sarcoma including adenosarcoma could occur. In malignant tumors, adenosarcoma is the second most common tumor after clear cell adenocarcinoma [8]. In addition, some studies have suggested that endometriosis has the potential to induce premalignant lesions [9]. Multiple pathways are involved in the malignant transformation and may share a common pathogenesis, the iron-induced oxidative stress due to repeated hemorrhage. It has been reported that persistent oxidative stress is associated with carcinogenesis [10]. Although not all adenosarcoma patients had a history of endometriosis, the majority cases of adenosarcoma arise from endometriosis. Therefore, the risk of malignant transformation might be significantly increased in the patients with extra-uterine endometriosis and recurrent and persistent disease stages. These patients are usually chemotherapy resistant. Close monitoring and follow-up are recommended. Moreover, endometriosis-associated tumors usually have a better outcome compared to the unassociated and mixed müllerian tumors [9]. 
MA is a low-grade and bi-directional tumor. Grossly, it is generally a polypoid, lobulated or papillary mass, with red-gray to tan appearance. Necrosis, hemorrhage and cyst cavity could be observed inside. Histologically, MA is composed of benign or atypical epithelial tissues that are mixed with sarcomatoid mesenchymal components. Benign endometriotic glands are embedded in a cellular stroma with polyp-like features, and it is similar to cystosarcoma phylloides of breast. The periglandular stromal cells are dense and nuclear atypia, ranged from low to moderate differentiation. The mitotic rate was above 4 per 10 HPFs. The "cuffing structure" is the most typical pathological feature of MA and has important implication in diagnosis. This cuffing structure is presented as the atypical hyperplasia of the stroma that surrounds the glandular components, mimicking a phenomenon of the overgrowth of the gland tissue. In addition, the interstitial edema lacking cell infiltration is often observed in the area distal to the center glands. The mesenchymal components of the MA are mostly homologous elements, such as smooth muscle, fibrous tissue and endometrial stroma, etc. Rarely, Heterologous elements are present. For example, the striated and cartilage muscle have been reported [11]. In 1989, Clement first described sarcomatous overgrowth in the MA and has defined the presence of pure sarcoma without any epithelial components. This type of sarcoma is accounted at least $25 \%$ [12] and is a high-grade and aggressive tumor compared to other adenosarcoma. Among the 6 cases of vaginal MA reported in the literature, 5 cases without sarcomatous overgrowth had a better outcome. Fortunately, our case is one of them.

As one of the adenosarcoma's clinical feature, nulligravida female patients are easily misdiagnosed. Therefore, postmenopausal or young woman who has experienced vaginal masses, rapidly enlarged uterine myoma and abdominal pain, adenosarcoma should be considered. The patient in our case had recurrent polyps in the vaginal stump and abdominal pain and irregular vaginal bleeding. She also had a history of endometriosis. Therefore, our case supports that adenosarcoma needs to be considered in these unusual clinical settings.

The standard treatment for the vaginal MA has not yet been established. Surgical removal, such as the cytoreductive surgery, followed by the adjuvant chemotherapy and/or radiotherapy is generally performed [13]. The five patients in the vaginal adenosarcoma cases reported in the literature, all underwent surgeries. Postoperatively, two patients had cisplatin-based chemotherapy and radiotherapy. One patient only had radiotherapy, but did have a good outcome. Another patient had received cisplatin-based chemocherapy, but she has already passed away. The fifth [5] patient had severe and refractory endometriosis. Her serum CA-125 level was abnormal during the endometriosis. This patient received radical operation that includes multiple surgical manipulations. Due to the high recurrence of her disease, three cycles of chemotherapy with the combined regimens of platinum, vincristine and bleomycin were conducted. However, recurred polyps in the vaginal stump with elevated CA-125 level (964.2 $\mathrm{U} / \mathrm{mL}$ ) were observed, which suggested a chemotherapy resistance. Therefore, 
the regimens were changed to PEI--cisplatin, epirubicin and ifosfamide, and total 9 cycles of chemotherapy with PEI was administered. After that, the patient took oral etoposide (VP16) as a maintaining treatment. The literature has reported other alternative treatments for the vaginal MA. Since low-grade adenosarcoma was positive for estrogen and progesterone receptors, progestin may have a therapeutic effect on adenosarcoma. Hines reported a case of extragenital adenosarcoma arising from endometriosis was treated by surgical removal of the lesions and followed by adjuvant therapy with medroxyprogesterone acetate (MPA) [14].

The patient in our case had the longest disease stage and the most complicated clinical features as well as the most severe endometriosis. Before the definitive diagnosis, she underwent repeated resections (6 times) of vaginal neoplasm and vaginal fistula repair due to the recurrent pelvic mass and vaginal cuff lesions. The treatment was not effective even after multiple consultations and various therapies. Finally, a combined multi-organ surgery was attempted. To avoid postoperative urinary and intestinal fistula, and to allow extensive surgical wounds to heal, delayed chemotherapy was recommended. The patient eventually refused to take the chemotherapy or radiotherapy, but was followed up regularly. She has not yet been found any evidence of recurrence for more than 9 years.

The behavior of malignant adenosarcoma seemed to be related to the grade of the malignant component, stromal mitotic figure and the tumor size. In our case, 1 - 2 stromal mitotic figures with mild nuclear atypia, about $4 \mathrm{~cm}$ mass and extensive resection procedure might indicate a good outcome without chemotherapy. To our knowledge, there are limited published data on the biological behavior and long-term clinical outcome of MA. As a result, it is necessary for the patient to be closely followed-up and periodically examined following treatment.

\section{Conclusion}

In conclusion, vaginal adenosarcoma is a neoplasm with low incidence and non-specific clinical features. Herein we present the first case of vaginal adenosarcoma without chemo-therapy or radiotherapy, and the patient is alive. Maybe it related to the size of the mass or thoroughness of the surgery. The histological characters of the tumor were typical, and the transition from endometriosis to ademosarcoma was gradual. Pathologically, vaginal adenosarcoma was a low-grade malignant tumor with a favorable prognosis compared to other types of uterine sarcoma. Immunohistochemistry was helpful in diagnosis and differential diagnosis. Due to the limited clinician's experiences and the variability of its clinical features, more cases need to be accumulated. These cases will be useful to define accurate diagnostic methods in the early stage and come up with effective treatments. If a young patient has been repeatedly received treatments for endometriosis, the doctor should be alert and adenosarcoma should be considered. 


\section{Consent}

Written informed consent was obtained from the patient for publication of this Case Report and any accompanying images. A copy of the written consent is available for review by the Editor-in-Chief of this journal.

\section{Acknowledgements}

We thank Mrs. Huang and Mrs. Bian for their technical assistance.

\section{Conflicts of Interest}

No author has any potential conflict of interest.

\section{References}

[1] Zhao, X.D. and Zhang, Y. (2003) Müllerian Adenosarcoma. Foreign Medical Fascicles of Gynecology and Obstetrics, 30, 231-236.

[2] Gallardo, A. and Prat, J. (2009) Müllerian Adenosarcoma: A Clinicopathologic and Immunohistochemical Study of 55 Cases Challenging the Existence of Adenofibroma. The American Journal of Surgical Pathology, 33, 278-288. https://doi.org/10.1097/PAS.0b013e318181a80d

[3] Judson, P.L. (2000) Vaginal Adenosarcoma Arising from Endometriosis. Gynecologic Oncology, 76, 123-125. https://doi.org/10.1006/gyno.1999.5617

[4] Anderson, J. (2001) Adenosarcoma in a Patient with Vaginal Endometriosis. Obstetrics \& Gynecology, 98, 964-966.

[5] Liu, L. and Davidson, S. (2003) Müllerian Adenosarcoma of Vagina Arising in Persistent Endometriosis: Report of a Case and Review of the Literature. Gynecologic Oncology, 90, 486-490. https://doi.org/10.1016/S0090-8258(03)00266-X

[6] Toyoshima, M., Akahira, J., Moriya, T., et al. (2004) Primary Vaginal Adenosarcoma with Sarcomatous Overgrowth. Gynecologic Oncology, 95, 759-761. https://doi.org/10.1016/j.ygyno.2004.08.004

[7] Han, X., Leng, J., Xiang, Y., et al. (2010) Vaginal Adenosarcoma Arising from Refractory Endometriosis: A Case Report. Aust N Z J Obstet Gynaecol, 50, 574-576.

[8] Dincer, A.D., Timmins, P., Pietrocola, D., et al. (2002) Primary Peritoneal Müllerian Adenosarcoma with Sarcomatous Overgrowth Associated with Endometriosis: A Case Report. International Journal of Gynecological Pathology, 21, 65-68. https://doi.org/10.1097/00004347-200201000-00012

[9] Al-Jefout, M., Tokushige, N., Hey-Cunningham, A.J., et al. (2009) Microanatomy and Function of the Eutopic Endometrium in Endometriosis. Expert Review of $O b$ stetrics \& Gynecology, 14, 61-79.

[10] Toyokuni, S. (2011) Mysterious Link between Iron Overload and CDKN2A/2B. Journal of Clinical Biochemistry and Nutrition, 48, 46-49. https://doi.org/10.3164/jcbn.11-001FR

[11] Bagga, R., Keepanasseril, A., Srinivasan, R., et al. (2010) Adenosarcoma of the Uterine Cervix with Heterologous Elements: A Case Report and Review of Literature. Archives of Gynecology and Obstetrics, 281, 669-675. https://doi.org/10.1007/s00404-009-1200-3

[12] Clement, P.B. (1989) Müllerian Adenosarcomas of the Uterus with Sarcomatous Overgrowth: A Clinical Pathological Analysis of 10 Cases. American Journal of Surgical Pathology, 13, 28-38. https://doi.org/10.1097/00000478-198901000-00004 
[13] Cantrell, L.A. and Van Le, L. (2009) Carcinosarcoma of the Ovary a Review. Obstetrical \& Gynecological Survey, 64, 673-80.

https://doi.org/10.1097/OGX.0b013e3181b8aff3

[14] Hines, B.J., Porges, R.F., Mittal, K., et al. (2002) Use of Medroxyprogesterone Acetate in the Treatment of Müllerian Adenosarcoma: A Case Report. Gynecologic Oncology, 85, 192-195. https://doi.org/10.1006/gyno.2002.6585 